\title{
The state of "freeze-for-all" in human ARTs
}

\author{
Natalia Basile $^{1}$ - Juan A. Garcia-Velasco ${ }^{1}$
}

Received: 31 May 2016/Accepted: 16 August 2016 / Published online: 14 September 2016

(C) Springer Science+Business Media New York 2016

\begin{abstract}
The recent development of vitrification technologies and the good outcomes obtained in assisted reproduction technologies have supported new indications for freezing and segmentation of treatment. Beyond OHSS prevention and avoidance of embryo transfers in the setting of an adverse endocrinological profile or endometrial cavity, we have witnessed a trend to shift fresh embryo transfers to frozen embryo transfers in many programs. We critically review the available evidence and suggest that freeze-all is not "for all," but should be individualized.
\end{abstract}

Keywords Vitrification · Freeze-all $\cdot$ OHSS $\cdot$ PGS $\cdot$ Low responders $\cdot$ Perinatal outcome $\cdot$ Segmentation

\section{Introduction}

Since the introduction of assisted reproduction technologies (ART) more than three decades ago, advances in clinical and laboratory areas have opened the door to different variants of the standard IVF procedure. The introduction of vitrification, safer stimulation protocols to prevent ovarian hyperstimulation syndrome (OHSS), increasing the utilization of preimplantation genetic testing, and data on obstetric and perinatal outcomes have led, among others, to a current state of

Capsule Segmentation of treatment with freeze-all and transfer in a subsequent cycle should not be routine, but only when indicated for a reason.

Juan A. Garcia-Velasco

juan.garcia.velasco@ivi.es

1 IVI-Madrid, Rey Juan Carlos University, Av del Talgo 68, 28023 Madrid, Spain
"Frozen Fever in ART." The elective deferred embryo transfer concept, based on the principle that controlled ovarian stimulation (COS) may cause a less physiological milieu for embryos to implant, is not uncommon these days. Yet, we must wonder when and for whom is this freeze-all approach most appropriate. Does it represent an opportunity to provide a more physiologically natural environment for the embryo: improving the soil? How can we balance this against the possible unnatural changes that we may be causing the embryo as a result of freezing: harming the seed? Physician's treatment choices are influenced by a number of factors, including guidelines, evidence-based medicine, past experience and, to a certain extent, habit [1]. This review attempts to describe what has driven part of the scientific community to shift from fresh to frozen embryo transfers, and to analyze if it is time to cool off and reconsider strategies.

\section{Freeze-all—why?}

\section{What has made us think of a possible shift to freeze-all policies?}

1. Evolution of cryopreservation techniques: toward vitrification.

Cryopreservation is of crucial importance in reproductive medicine. Since the mid-1980s, cryopreservation and storage of embryos created in vitro has been employed to ensure that most of the potential of an IVF cycle is made available to patients without the need for the simultaneous transfer of multiple embryos. Slow freezing has been the method of choice for decades for the cryopreservation of cleavage-stage embryos and blastocysts. In oocyte terms, however, although a number of pregnancies and births have been reported following the transfer of embryos 
derived from slow-freezed oocytes [2-4], survival rates are only close to $50 \%$ [5-8] and fertilization rates of frozenthawed oocytes are low compared to fresh ones (just over $50 \%$ overall) [9]. Vitrification, on the other hand, has proven more effective than slow freezing in terms of survival rates and clinical outcomes for oocytes and embryos [10-12], and no doubt is the method of choice in practically every IVF clinic today. Vitrification is a fast cryopreservation method that involves exposure of oocytes/ embryos at high concentrations of cryoprotectants and ultra-rapid cooling rates which cause instant solidification (vitrous state) that avoids damage to the cell due to ice crystal formation. The avoidance of ice crystal formation represents the major advantage of vitrification over slow freezing. Given the excellent results provided by this technique, vitrification can no longer be taken as a complement to IVF treatments but as a fundamental tool for the management of our patients. Low responders, for instance, can benefit from accumulation of oocytes through vitrification as it allows them to achieve similar clinical outcomes as normo-responders [13]. Moreover, this strategy is also attractive for patients of advanced maternal age (a more difficult group to treat) who, in addition to being low responders, require preimplantation genetic screening [14]. On the contrary, vitrification of oocytes (and embryos) has also proven to work with patients at risk of developing OHSS and represents a solution when embryo transfer needs to be delayed [15, 16]. Last but not least, oocyte vitrification allows women to preserve their fertility today, the so-called "social freezing," and offers clinics to establish egg donor banks with similar results to fresh oocytes [17]. In terms of live birth rates, a population-based cohort study that compared cryopreservation by vitrification or slow freezing in a large data set (over 30,000 cases between the two groups) revealed that vitrified blastocyst transfer cycles resulted in a significantly higher live delivery rate (adjusted relative risk (ARR) 1.41, 95\%CI 1.341.49) compared with slow frozen blastocyst transfer cycles. One limitation of this study is the lack of information available on clinic-specific cryopreservation protocols and processes for slow freezing / thawing and vitrification / warming of blastocysts and the potential impact on outcomes [18]. Thus, introducing vitrification has been crucial, and it today represents an excellent tool when it comes to establishing freeze-all policies in our clinics.

2. Improvements in stimulation protocols: toward antagonist protocols and OHSS-free clinics.

Controlled ovarian stimulation is the initial step of an IVF treatment that has evolved from simple protocols to personalized treatments. The use of oral contraceptive pills prior to ovarian stimulation was an early step toward programming IVF cycles [19, 20], but later, the introduction of gonadotropin-releasing hormone agonists $(\mathrm{GnRH}$ agonists) led to a major change in terms of IVF logistics. GnRH agonists are widely used to downregulate endogenous FSH and LH secretion, to prevent premature endogenous luteinizing hormone (LH) surge and to reduce cancelations due to luteinization [21, 22]. For many years, GnRH agonists have been considered the "gold standard" protocol, especially in young normo-responders. Yet, one major drawback of these protocols is the risk of OHSS. The introduction of GnRH antagonists seemed to open up a new way to more "friendly IVF" [23]. Unlike indirect pituitary suppression induced by GnRH agonists, administration of $\mathrm{GnRH}$ antagonists causes immediate and dose-related suppression of gonadotropin release by competitive occupancy of $\mathrm{GnRH}$ receptors in the pituitary [24], which results in a much shorter duration of stimulation and absence of side effects caused by profound hypoestrogenemia $[25,26]$. A meta-analysis by AlInany et al. demonstrated that the live birth rates between the use of GnRH antagonists and agonist protocols were comparable [27], but the majority of trials clearly support $\mathrm{GnRH}$ antagonists resulting in a significantly lower incidence $(50 \%)$ of OHSS compared with GnRH agonists [27-29]. Following the introduction of the antagonist protocols, complementary measures came along to avoid the risk of OHSS even more, like triggering ovulation induction with GnRH agonists instead of human chorionic gonadotropin (HCG) [30-33]. Still, this approach is not $100 \%$ safe as a few anecdotal cases of severe OHSS following GnRH agonist triggering with no luteal supplementation have been reported [34-36]. GnRH agonist triggering has been associated with luteal phase defects, presumably due to excessive negative steroid feedback [37-39]. Two consecutive Cochrane reviews by the same author state that: "GnRH agonist triggering is associated with a lower ongoing pregnancy rate compared with conventional hCG" $[29,40]$. This conclusion was qualified as premature and mistaken based on the following: (1) Cochrane reviews should not be conducted in the research phase when new concepts are being developed, and (2) the authors missed the fact that the variable that affects results is luteal phase support itself and not the use of GnRH agonist trigger for final oocyte maturation [39]. Therefore, if triggering with $\mathrm{GnRH}$ agonist (which is the preferred strategy to reduce the risk of OHSS) is associated with luteal phase defects, then a fresh embryo transfer should not take place; treatment needs to be segmented and the freeze-all concept becomes attractive.

3. Inevitable increase in genetic screening: toward the transfer of a healthy embryo.

Despite ongoing debate about its efficacy, preimplantation genetic screening (PGS) is increasingly being used to improve implantation rates after IVF. The European Society of Human Reproduction and 
Embryology (ESHRE) PGD Consortium collects preimplantation genetic diagnosis data (PGD) from a number of clinics worldwide. According to its last report, $52 \%$ of the PGD cycles in 2010 were used as PGS [41]. In early stages, PGD was used to detect embryos at risk of inheriting monogenic diseases. In 1990, Handyside et al. [42] treated couples with sex-linked disorders through DNA amplification followed by PCR for a specific repeated gene sequence on the Y chromosome. Later, PCR was found to be less effective in determining gender as it produces qualitative and non-quantitative data, which means that test results could confirm sex by highlighting the presence or absence of a specific repeated gene sequence unique to the $\mathrm{Y}$ chromosome without providing further information on the existence of an $\mathrm{X}$ or $\mathrm{Y}$ chromosome [43]. To overcome this disadvantage, fluorescent in situ hybridization (FISH) came along and expanded rapidly to also include somatic chromosomes [44]. Considering the well-known fact that chromosomal abnormalities play a major role in failed embryo implantation, it was not surprising to hypothesize that identifying aneuploid embryos in patients without a history of genetic disorders could improve implantation rates and live birth, while reducing the number of abortions at the same time. This is how preimplantation genetic screening (PGS) started, and today, we have evolved from the early FISH years to the use of array $\mathrm{CGH}$ and next-generation sequencing (NGS). The commonest indications for PGS in IVF include the following: patients of advanced maternal age (AMA), repeated implantation failure (RIF), recurrent pregnancy loss (RPL), and, to a lesser extent, patients with partners with male factor infertility [45, 46] and egg donors [47]. From a technical point of view, genetic screening has evolved from biopsies of the oocyte's first and second polar bodies $[48,49]$ to biopsies of cleavage-stage embryos. The first clinical studies conducted with biopsies on cleavage-stage embryos showed higher implantation and ongoing pregnancy rates, as well as lower abortion rates $[50,51]$. The initial belief about day 3 biopsy was that it gave the laboratory plenty of time to do the actual testing and still be able to do a fresh embryo transfer on day 5 . However, there were several drawbacks, including mosaicism and embryo damage due to the removal of a large percentage of embryo cells [52]. Fortunately, advancements in culture media formulations allowed embryologists to culture embryos to later development stages and the first cases of trophectoderm biopsy took place in 2005 [53]. Today, trophectoderm biopsy is the method of choice not only for a reduced likelihood of mosaicism but also to increase the amount of DNA available for testing and to cause less embryo damage. It is in this context that the freeze-all idea plays a role by overcoming time restraints and allowing us to biopsy embryos in later development stages, vitrify them immediately afterward, send the cells to be tested, wait for the results to come back, and schedule the transfer of a normal embryo in a subsequent cycle.

4. Data on perinatal and obstetric outcomes: toward safer mothers and offspring.

There is a growing body of evidence that highlights concerns about perinatal and obstetric outcomes after ART, particularly following fresh transfers.

Different studies have shown that children born after assisted reproductive techniques (ART) have poorer outcomes in terms of preterm birth (PTB), low birth weight (LBW), small for gestational age (SGA), and perinatal mortality when compared with newborns of natural conceptions [54-56]. Among those undergoing IVF, the results between fresh and frozen embryo transfers (FET) in obstetric perinatal outcome terms are still unclear [57-59]. A systematic review and meta-analysis based on $11 \mathrm{ob}-$ servational studies concluded that after transferring frozen-thawed embryos, singleton pregnancies resulted in a lower risk of antepartum hemorrhage $(\mathrm{RR}=0.67$, 95\%CI 0.55-0.81), PTB ( $\mathrm{RR}=0.84,95 \%$ CI $0.78-0.90)$, SGA $(\mathrm{RR}=0.45,95 \% \mathrm{CI} 0.30-0.66), \mathrm{LBW}(\mathrm{RR}=0.69$, $95 \% \mathrm{CI} 0.62-0.76)$, and perinatal mortality $(\mathrm{RR}=0.68$, 95\%CI 0.48-0.96) than with fresh embryos [60]. A Scandinavian study that included three different countries [58] corroborated some of these findings, but also observed that frozen-thawed embryos were at higher risk of post-term birth (aOR 1.40, 95\%CI 1.27-1.55), large for gestacional age (LGA) (aOR 1.45, 95\%CI 1.271.64), macrosomia (aOR 1.58, 95\%CI 1.39-1.80) and perinatal mortality (aOR 1.49, 95\%CI 1.07-2.07), which was in line with other large population-based studies [61, 62]. More recent evidence from another large observational study in Australia [63] has confirmed that embryo transfers of vitrified-warmed blastocysts yield equivalent live birth rates than fresh blastocysts, but significantly improved neonatal outcomes in terms of gestational age, being 0.3 weeks longer on average, and for live birth weight with babies born $145 \mathrm{~g}$ heavier on average compared with fresh transfers. However, we may wonder what the clinical relevance of all this actually is. Finally, this year, a report from a group in Brussels analyzed 1072 singletons and twins born after embryo vitrification on day 3 and day 5 and concluded that neonatal health parameters, including the prevalence of congenital malformations, were similar to or slightly better than after fresh embryo transfer [64]. It should be acknowledged that the majority of these studies are observational and that vitrified embryos are always second choice for transfer. We could assume that elective frozen embryo transfer with first choice embryos could yield even better results, but these studies are yet to be performed. Therefore, 
published data suggest that it is still unclear if perinatal and obstetric outcomes are better with FETs compared to fresh ones (Table 1).

\section{Freeze-all for whom?}

Despite all the abovementioned benefits, we must still wonder who should benefit from the freeze-all policy. The debate perhaps arises as a result of a recent meta-analysis [65], which concluded that: "The use of FET, compared with fresh embryo transfer, significantly improves clinical and ongoing pregnancy rates in patients submitted to ART." However, this conclusion is highly questionable because of different factors:

First, the meta-analysis includes only three small studies. A proper meta-analysis should involve at least 3-4 RCTs with no less than 1000 participants [66]. This was not met in this review as the total number of patients included was only 633 . In addition, one of the studies [67], in fact the largest one, was retracted at the request of the Editor and the ASRM Publications Committee due to serious methodological flaws, which reduced the total number even more to back up such a conclusion. Second, the three studies are very heterogeneous in terms of the types of patients included (normo- and high responders) and of some technical aspects. Normo- and high responders represent two different entities in terms of clinical approach, management, and IVF outcomes, not to mention safety issues, which differ very much from one group of patients to another. In addition, the cryopreservation technique and the development stage in which embryos were cryopreserved differed. Both studies by Shapiro et al. [68, 69] performed the slow freezing of 2PNs, whereas Aflatoonian et al. did vitrification on day 2 of development. Third, the study by Shapiro et al. [69], which included normo-responders was underpowered (the required sample size was 411 patients and the study randomized only 67 patients for fresh transfer and 70 for FET), involved co-interventions, such as dual trigger for final oocyte maturation, and led to pregnancy rates of $84 \%$ in the FET group, which were far higher than those reported in European or American registries. Fourth, none of these RCTs were able to provide data on live birth rates, costeffectiveness, and acceptability from patients.

In our opinion, there is no doubt that patients at risk of OHSS are good candidates for this approach. For these patients, the combination of the right stimulation protocol and the vitrification of oocytes/embryos have transformed what was a high-risk situation into an anecdotic one [70, 71]. As described by Devroey et al. [72], the strategy to obtain OHSSfree clinics is closely related to the segmentation concept. It consists of the following: segment A, stimulation with GnRH antagonist and triggering with $\mathrm{GnRH}$ agonist; segment $\mathrm{B}$, optimum cryopreservation of oocytes or embryos through vitrification; and segment $\mathrm{C}$, embryo replacement into a receptive, non stimulated endometrium in a natural cycle or with artificial endometrial preparation. Regarding segment B, one common question is whether if we should vitrify oocytes or embryos. According to a prospective comparison made by Herrero et al., both approaches are safe as they result in similar ongoing pregnancy rates and live births after three transfers [15]. Therefore, the decision to do one or the other will most likely depend on the individual patient's preferences, and on ethical and cultural issues rather than on clinical outcomes. A trial of expectations and perceptions of frozen embryo holders revealed that many couples are concerned about frozen embryo disposition for ethical reasons [73]. Given these concerns, oocyte vitrification may ultimately be a more attractive and realistic alternative for certain society groups [74]. As regards segment $\mathrm{C}$, no consensus has been reached on the most effective endometrium preparation method prior to FET. A systematic review and meta-analysis published in 2013 compared clinical and ongoing pregnancy rates, as well as live birth rates, in (a) true natural cycle FET (NC-FET), (b) modified NC-FET with hCG, (c) artificial cycle FET (ACFET), and (d) AC-FET supplemented with GnRH agonists. The study revealed no significant advantage of any specific approach to prepare the endometrium for FET in terms of clinical pregnancy rates or live birth rates [75]. The results were in line with the previous systematic review published in 2008 [76]. Either way, and independently of the particular characteristics of segments $\mathrm{B}$ and $\mathrm{C}$, there is no doubt that the segmentation approach for patients with OHSS works [70, 71,
Table 1 Perinatal and obstetric outcomes of frozen embryo transfers

\begin{tabular}{lll}
\hline Reduced risks in FET & Increased risks with FET & Unclear \\
\hline LBW $(<2500 \mathrm{~g})$ & Macrosomia $(>4500 \mathrm{~g})$ & Ectopic pregnancy \\
SGA $(<10 \%)$ & Large for GA & Pre-eclampsia \\
Placental abruption & Placenta accreta & Congenital abnormalities \\
Placenta previa & C-section delivery & NICU \\
Antepartum hemorrhage & & VLBW $(<1500 \mathrm{~g})$ \\
Perinatal mortality & & VPTB $(<32$ weeks $)$ \\
\hline
\end{tabular}

$L B W$ low birth weight, $S G A$ small for gestational age, $G A$ gestational age, NICU neonatal intensive care unit, $V L B W$ very low birth weight, $V P T B$ very preterm birth 
$77,78]$, and that the freeze-all policy in this situation is no longer controversial.

However, controversy still exists when it comes to normoresponders. The benefits of the freeze-all policy for patients at risk of OHSS cannot be extrapolated to safe normo-responders. A recent study by our group [79] revealed no difference in terms of ongoing pregnancy and live birth rates between normo-responder patients who underwent fresh and deferred embryo transfers. This was a retrospective study that included 882 patients aged 20-44 who underwent their first or second IVF/ICSI cycle, in which a normal oocyte yield [4-20] was obtained. Patients were divided into two groups: 364 (41.3\%) underwent fresh embryo transfer and 518 (58.7\%) were submitted to freeze-all, according to their doctor's preference. No differences were observed between both patient groups in terms of demographic characteristics and ovarian stimulation parameters. The raw analysis showed that ongoing pregnancy ( 36.2 vs. $33.8 \%, p=0.51)$ and live birth (33.2 vs. $32.9 \%, p=0.94)$ rates for freeze-all vs. fresh cycles, respectively, were similar. When adjustments were made for patient age and other variables likely to affect the results, there was still no evidence for any impact of freezing. All the patients in the study were considered "normal responders" and were thus representative of a routine IVF patient population. Unlike these results, a different study by Roque et al. [80] found better ongoing pregnancy rates using the freeze-all policy in patients with progesterone levels $\leq 1.5 \mathrm{ng} / \mathrm{mL}$ on the day of triggering. However, sample size was smaller than our study (530 patients: 351 in the fresh group and 179 in the FET group). In addition, the retrospective design of this study does not allow us to understand if poor responders were transferred, and if normo- and high responders were frozen, which would have biased the results.

It should be acknowledged that a normal response per se is not exclusive of freeze-all. Normo-responders can face situations in which this policy may apply, such as those who undergo genetic screening, or present high progesterone levels, impaired endometrium, etc. If it is not indicated, the question whether freeze-all represents a benefit for the general IVF population still remains, and further well-designed prospective studies will answer this question. This is the case of an ongoing randomized controlled trial that is taking place in the UK (EFREEZE trial). In this multicentric study, 1086 women (543 in each arm) will be randomized over a 2-year period to show (or not) an absolute difference in the primary outcome of at least $9 \%$ (with $90 \%$ power and a two-sided $5 \%$ level of statistical significance). The primary outcome has been defined as follows: a live singleton baby born at term with appropriate weight for gestation (AGA). A composite primary outcome has been chosen to reflect safety (single, term, and AGA) and effectiveness (live birth) since the goal of modern IVF is accepted to be a healthy baby and a healthy mother (with no OHSS) (http://www.nets.nihr.ac.uk/projects/hta/1311582).
Freeze-all-conclusion-change in practice or not?

A change in practice should be based on good evidence and sufficiently powered studies. As of today, the benefits of the freeze-all policy are based mainly on high responder patients and cannot be extrapolated to the general IVF population. We are entering the era of individualized treatments in IVF, not only from a clinical point of view, but also from the laboratory technologies that we offer our patients. Why would the freezeall policy be exempt from this individualization, especially since there is not enough data to justify it?

Clear indications should be specified (Table 2). In our center, indications for freeze-all are not only restricted to patients at risk of OHSS but also to any type of patient with a clear indication: patients seeking genetic screening, patients with an altered endocrine and cardiovascular profile at the time of transfer (elevated progesterone, hypertension, etc.), patients with inadequate uterine cavity for embryo transfer (i.e., fluid in cavity), and low responders who seek a strategy to accumulate oocytes or embryos. In some areas around the world, especially Latin America, the endemic situation of the Zika virus is causing patients to either postpone or interrupt their IVF treatments to avoid infection during pregnancy. However, postponing is not a choice for some of our patients since they are already fighting against their biological clock. Therefore, initiating treatment and freezing all the embryos represent a solution.

The importance of a good laboratory should be emphasized. Implementation of the freeze-all policy will not work unless high survival rates for oocytes and embryos are guaranteed. This aspect is not minor and is the essence for this policy to work. Any embryologist would agree that vitrification is a laborious process that requires plenty of focus and is inevitably affected by a long learning curve. In this context, perhaps the introduction of automatic systems could help the standardization of results and make the implementation of freeze-all policies easier. Finally, what about the extra cost and time that it would represent? Would IVF patients accept this shift? Are we ready to handle their expectations? A study that evaluated patient attitudes toward elective single embryo transfer (eSET) clearly showed that even though informed

Table 2 Indications for frozen embryo transfers

FET indications

\section{OHSS}

Elevated progesterone

Genetic Screening

Low responders

Inadequate uterine cavity

Hypertension

Zika virus (areas affected) 
patients are sufficiently concerned about the adverse outcomes of multiple pregnancies, they become less risk-concerned when faced with multiple IVF failures and economic burden [73]. Then the question is - why would elective freezing be any different from elective single embryo transfer? Enough questions still need to be answered to justify the implementation of the freeze-all policy when there is no real indication. Therefore, it is time to cool off, wait for more evidence, and shift only if necessary.

\section{References}

1. Lode H, Torres A, Cockle A. What drives our choices? Evidence, guidelines or habit? Int J Antimicrob Agents. 2007;29 Suppl 1:S1722.

2. Tucker MJ, Wright G, Morton PC, Massey JB. Birth after cryopreservation of immature oocytes with subsequent in vitro maturation. Fertil Steril. 1998;70:578-9.

3. Borini A, Bonu MA, Coticchio G, Bianchi V, Cattoli M, Flamigni C. Pregnancies and births after oocyte cryopreservation. Fertil Steril. 2004;82:601-5.

4. De Santis L, Cino I, Rabellotti E, Papaleo E, Calzi F, Fusi FM, et al. Oocyte cryopreservation: clinical outcome of slow-cooling protocols differing in sucrose concentration. Reprod Biomed Online. 2007;14:57-63.

5. Porcu E, Fabbri R, Seracchioli R, Ciotti PM, Magrini O, Flamigni C. Birth of a healthy female after intracytoplasmic sperm injection of cryopreserved human oocytes. Fertil Steril. 1997;68:724-6.

6. Chia CM, Chan WB, Quah E, Cheng LC. Triploid pregnancy after ICSI of frozen testicular spermatozoa into cryopreserved human oocytes: case report. Hum Reprod. 2000;15:1962-4.

7. Miller KA, Elkind-Hirsch K, Levy B, Graubert MD, Ross SJ, Scott Jr RT. Pregnancy after cryopreservation of donor oocytes and preimplantation genetic diagnosis of embryos in a patient with ovarian failure. Fertil Steril. 2004;82:211-4.

8. Greco E, Iacobelli M, Rienzi L, Fabris GF, Tesorio N, Tesarik J. Birth of a healthy boy after fertilization of cryopreserved oocytes with cryopreserved testicular spermatozoa from a man with nonmosaic Klinefelter syndrome. Fertil Steril. 2008;89:991.e5-7.

9. Gook DA, Edgar DH. Human oocyte cryopreservation. Hum Reprod Update. 2007;13:591-605.

10. Rezazadeh Valojerdi M, Eftekhari-Yazdi P, Karimian L, Hassani F, Movaghar B. Vitrification versus slow freezing gives excellent survival, post warming embryo morphology and pregnancy outcomes for human cleaved embryos. J Assist Reprod Genet. 2009;26:347-54.

11. Stehlik E, Stehlik J, Katayama KP, Kuwayama M, Jambor V, Brohammer R, et al. Vitrification demonstrates significant improvement versus slow freezing of human blastocysts. Reprod Biomed Online. 2005;11:53-7.

12. Cobo A, Perez S, De los Santos MJ, Zulategui J, Domingo J, Remohi J. Effect of different cryopreservation protocols on the metaphase II spindle in human oocytes. Reprod Biomed Online. 2008; 17:350-9.

13. Cobo A, Garrido N, Crespo J, Jose R, Pellicer A. Accumulation of oocytes: a new strategy for managing low-responder patients. Reprod Biomed Online. 2012;24:424-32.

14. Milan M, Cobo AC, Rodrigo L, Mateu E, Mercader A, Buendia P, et al. Redefining advanced maternal age as an indication for preimplantation genetic screening. Reprod Biomed Online. 2010;21:649-57.

15. Herrero L, Pareja S, Aragones M, Cobo A, Bronet F, GarciaVelasco JA. Oocyte versus embryo vitrification for delayed embryo transfer: an observational study. Reprod Biomed Online. 2014;29: 567-72.

16. Herrero L, Pareja S, Losada C, Cobo AC, Pellicer A, GarciaVelasco JA. Avoiding the use of human chorionic gonadotropin combined with oocyte vitrification and GnRH agonist triggering versus coasting: a new strategy to avoid ovarian hyperstimulation syndrome. Fertil Steril. 2011;95:1137-40.

17. Cobo A, Kuwayama M, Perez S, Ruiz A, Pellicer A, Remohi J. Comparison of concomitant outcome achieved with fresh and cryopreserved donor oocytes vitrified by the Cryotop method. Fertil Steril. 2008;89:1657-64.

18. Li Z, Wang YA, Ledger W, Edgar DH, Sullivan EA. Clinical outcomes following cryopreservation of blastocysts by vitrification or slow freezing: a population-based cohort study. Hum Reprod. 2014;29:2794-801.

19. Frydman R, Forman R, Rainhorn JD, Belaisch-Allart J, Hazout A, Testart J. A new approach to follicular stimulation for in vitro fertilization: programmed oocyte retrieval. Fertil Steril. 1986;46:657-62.

20. Wardle PG, Foster PA, Mitchell JD, McLaughlin EA, Williams JA, Corrigan E, et al. Norethisterone treatment to control timing of the IVF cycle. Hum Reprod. 1986;1:455-7.

21. Stanger JD, Yovich JL. Reduced in-vitro fertilization of human oocytes from patients with raised basal luteinizing hormone levels during the follicular phase. Br J Obstet Gynaecol. 1985;92:385-93.

22. Wildt L, Diedrich K, van der Ven H, al Hasani S, Hubner H, Klasen R. Ovarian hyperstimulation for in-vitro fertilization controlled by GnRH agonist administered in combination with human menopausal gonadotrophins. Hum Reprod. 1986;1:15-9.

23. Olivennes F, Frydman R. Friendly IVF: the way of the future? Hum Reprod. 1998;13:1121-4.

24. Huirne JA, Homburg R, Lambalk CB. Are GnRH antagonists comparable to agonists for use in IVF? Hum Reprod. 2007;22:2805-13.

25. Borm G, Mannaerts B. Treatment with the gonadotrophin-releasing hormone antagonist ganirelix in women undergoing ovarian stimulation with recombinant follicle stimulating hormone is effective, safe and convenient: results of a controlled, randomized, multicentre trial. The European Orgalutran Study Group. Hum Reprod. 2000;15: 1490-8.

26. Fluker M, Grifo J, Leader A, Levy M, Meldrum D, Muasher SJ, et al. Efficacy and safety of ganirelix acetate versus leuprolide acetate in women undergoing controlled ovarian hyperstimulation. Fertil Steril. 2001;75:38-45.

27. Al-Inany HG, Youssef MA, Aboulghar M, Broekmans F, Sterrenburg M, Smit J, et al. Gonadotrophin-releasing hormone antagonists for assisted reproductive technology. Cochrane Database Syst Rev 2011;(5):CD001750. doi:CD001750.

28. Tarlatzis BC, Kolibianakis EM. GnRH agonists vs antagonists. Best Pract Res Clin Obstet Gynaecol. 2007;21:57-65.

29. Youssef MA, Van der Veen F, Al-Inany HG, Griesinger G, Mochtar $\mathrm{MH}$, Aboulfoutouh I, et al. Gonadotropin-releasing hormone agonist versus HCG for oocyte triggering in antagonist assisted reproductive technology cycles. Cochrane Database Syst Rev 2011;(1): CD008046. doi:CD008046.

30. Kolibianakis EM, Schultze-Mosgau A, Schroer A, van Steirteghem A, Devroey P, Diedrich K, et al. A lower ongoing pregnancy rate can be expected when GnRH agonist is used for triggering final oocyte maturation instead of HCG in patients undergoing IVF with GnRH antagonists. Hum Reprod. 2005;20:2887-92.

31. Kolibianakis EM, Tarlatzis B, Devroey P. GnRH antagonists in IVF. Reprod Biomed Online. 2005;10:705-12.

32. Griesinger G, Diedrich K, Devroey P, Kolibianakis EM. GnRH agonist for triggering final oocyte maturation in the $\mathrm{GnRH}$ 
antagonist ovarian hyperstimulation protocol: a systematic review and meta-analysis. Hum Reprod Update. 2006;12:159-68.

33. Youssef MA, Van der Veen F, Al-Inany HG, Griesinger G, Mochtar $\mathrm{MH}$, van Wely M. Gonadotropin-releasing hormone agonist versus HCG for oocyte triggering in antagonist assisted reproductive technology cycles. Cochrane Database Syst Rev 2010;(11):CD008046. doi:CD008046.

34. Fatemi HM, Popovic-Todorovic B, Humaidan P, Kol S, Banker M, Devroey P, et al. Severe ovarian hyperstimulation syndrome after gonadotropin-releasing hormone $(\mathrm{GnRH})$ agonist trigger and "freeze-all" approach in GnRH antagonist protocol. Fertil Steril. 2014;101:1008-11.

35. Gurbuz AS, Gode F, Ozcimen N, Isik AZ. Gonadotrophin-releasing hormone agonist trigger and freeze-all strategy does not prevent severe ovarian hyperstimulation syndrome: a report of three cases. Reprod Biomed Online. 2014;29:541-4.

36. Santos-Ribeiro S, Polyzos NP, Stouffs K, De Vos M, Seneca S, Tournaye $\mathrm{H}$, et al. Ovarian hyperstimulation syndrome after gonadotropin-releasing hormone agonist triggering and "freezeall": in-depth analysis of genetic predisposition. J Assist Reprod Genet. 2015;32:1063-8.

37. Segal S, Casper RF. Progesterone supplementation increases luteal phase endometrial thickness and oestradiol levels in in-vitro fertilization. Hum Reprod. 1992;7:1210-3.

38. Casper RF. Introduction: gonadotropin-releasing hormone agonist triggering of final follicular maturation for in vitro fertilization. Fertil Steril. 2015;103:865-6.

39. Kol S, Humaidan P, Alsbjerg B, Engmann L, Benadiva C, GarciaVelasco JA, et al. The updated Cochrane review 2014 on GnRH agonist trigger: repeating the same errors. Reprod Biomed Online. 2015;30:563-5.

40. Youssef MA, Van der Veen F, Al-Inany HG, Mochtar MH, Griesinger G, Nagi Mohesen M, et al. Gonadotropin-releasing hormone agonist versus HCG for oocyte triggering in antagonistassisted reproductive technology. Cochrane Database Syst Rev. 2014;10:CD008046.

41. De Rycke M, Belva F, Goossens V, Moutou C, SenGupta SB, Traeger-Synodinos J, et al. ESHRE PGD Consortium data collection XIII: cycles from January to December 2010 with pregnancy follow-up to October 2011. Hum Reprod. 2015;30:1763-89.

42. Handyside AH, Kontogianni EH, Hardy K, Winston RM. Pregnancies from biopsied human preimplantation embryos sexed by Y-specific DNA amplification. Nature. 1990;344:768-70.

43. Ly KD, Agarwal A, Nagy ZP. Preimplantation genetic screening: does it help or hinder IVF treatment and what is the role of the embryo? J Assist Reprod Genet. 2011;28:833-49.

44. Munne S, Weier HU, Stein J, Grifo J, Cohen J. A fast and efficient method for simultaneous $\mathrm{X}$ and $\mathrm{Y}$ in situ hybridization of human blastomeres. J Assist Reprod Genet. 1993;10:82-90.

45. Silber S, Escudero T, Lenahan K, Abdelhadi I, Kilani Z, Munne S. Chromosomal abnormalities in embryos derived from testicular sperm extraction. Fertil Steril. 2003;79:30-8.

46. Platteau P, Staessen C, Michiels A, Tournaye H, Van Steirteghem A, Liebaers I, et al. Comparison of the aneuploidy frequency in embryos derived from testicular sperm extraction in obstructive and nonobstructive azoospermic men. Hum Reprod. 2004;19:1570-4.

47. Munne S, Fischer J, Warner A, Chen S, Zouves C, Cohen J, et al. Preimplantation genetic diagnosis significantly reduces pregnancy loss in infertile couples: a multicenter study. Fertil Steril. 2006;85: 326-32.

48. Munne S, Dailey T, Sultan KM, Grifo J, Cohen J. The use of first polar bodies for preimplantation diagnosis of aneuploidy. Hum Reprod. 1995;10:1014-20.

49. Verlinsky Y, Kuliev A. Preimplantation polar body diagnosis. Biochem Mol Med. 1996;58:13-7.
50. Munne S, Chen S, Fischer J, Colls P, Zheng X, Stevens J, et al. Preimplantation genetic diagnosis reduces pregnancy loss in women aged 35 years and older with a history of recurrent miscarriages. Fertil Steril. 2005;84:331-5.

51. Gianaroli L, Magli MC, Ferraretti AP, Munne S. Preimplantation diagnosis for aneuploidies in patients undergoing in vitro fertilization with a poor prognosis: identification of the categories for which it should be proposed. Fertil Steril. 1999;72:837-44.

52. Cohen J, Wells D, Munne S. Removal of 2 cells from cleavage stage embryos is likely to reduce the efficacy of chromosomal tests that are used to enhance implantation rates. Fertil Steril. 2007;87:496-503.

53. McArthur SJ, Leigh D, Marshall JT, de Boer KA, Jansen RP. Pregnancies and live births after trophectoderm biopsy and preimplantation genetic testing of human blastocysts. Fertil Steril. 2005;84:1628-36.

54. Helmerhorst FM, Perquin DA, Donker D, Keirse MJ. Perinatal outcome of singletons and twins after assisted conception: a systematic review of controlled studies. BMJ. 2004;328:261.

55. Henningsen AK, Pinborg A, Lidegaard O, Vestergaard C, Forman $\mathrm{JL}$, Andersen AN. Perinatal outcome of singleton siblings born after assisted reproductive technology and spontaneous conception: Danish national sibling-cohort study. Fertil Steril. 2011;95:959-63.

56. Bloise E, Feuer SK, Rinaudo PF. Comparative intrauterine development and placental function of ART concepti: implications for human reproductive medicine and animal breeding. Hum Reprod Update. 2014;20:822-39.

57. Pelkonen S, Koivunen R, Gissler M, Nuojua-Huttunen S, Suikkari AM, Hyden-Granskog C, et al. Perinatal outcome of children born after frozen and fresh embryo transfer: the Finnish cohort study 1995-2006. Hum Reprod. 2010;25:914-23.

58. Wennerholm UB, Henningsen AK, Romundstad LB, Bergh C, Pinborg A, Skjaerven R, et al. Perinatal outcomes of children born after frozen-thawed embryo transfer: a Nordic cohort study from the CoNARTaS group. Hum Reprod. 2013;28:2545-53.

59. Ishihara O, Araki R, Kuwahara A, Itakura A, Saito H, Adamson GD. Impact of frozen-thawed single-blastocyst transfer on maternal and neonatal outcome: an analysis of 277,042 single-embryo transfer cycles from 2008 to 2010 in Japan. Fertil Steril. 2014;101:128-33.

60. Maheshwari A, Pandey S, Shetty A, Hamilton M, Bhattacharya S. Obstetric and perinatal outcomes in singleton pregnancies resulting from the transfer of frozen thawed versus fresh embryos generated through in vitro fertilization treatment: a systematic review and meta-analysis. Fertil Steril 2012;98:368,77.e1-9.

61. Pinborg A, Loft A, Aaris Henningsen AK, Rasmussen S, Andersen AN. Infant outcome of 957 singletons born after frozen embryo replacement: the Danish National Cohort Study 1995-2006. Fertil Steril. 2010;94:1320-7.

62. Sazonova A, Kallen K, Thurin-Kjellberg A, Wennerholm UB, Bergh C. Obstetric outcome in singletons after in vitro fertilization with cryopreserved/thawed embryos. Hum Reprod. 2012;27:1343-50.

63. Roy TK, Bradley CK, Bowman MC, McArthur SJ. Single-embryo transfer of vitrified-warmed blastocysts yields equivalent live-birth rates and improved neonatal outcomes compared with fresh transfers. Fertil Steril. 2014;101:1294-301.

64. Belva F, Bonduelle M, Roelants M, Verheyen G, Van Landuyt L. Neonatal health including congenital malformation risk of 1072 children born after vitrified embryo transfer. Hum Reprod 2016.

65. Roque M, Lattes K, Serra S, Sola I, Geber S, Carreras R, et al. Fresh embryo transfer versus frozen embryo transfer in in vitro fertilization cycles: a systematic review and meta-analysis. Fertil Steril. 2013;99:156-62.

66. Humaidan P, Polyzos NP. (Meta)analyze this: systematic reviews might lose credibility. Nat Med. 2012;18:1321.

67. Aflatoonian A, Oskouian H, Ahmadi S, Oskouian L. Can fresh embryo transfers be replaced by cryopreserved-thawed embryo 
transfers in assisted reproductive cycles? A randomized controlled trial. J Assist Reprod Genet. 2010;27:357-63.

68. Shapiro BS, Daneshmand ST, Garner FC, Aguirre M, Hudson C, Thomas S. Evidence of impaired endometrial receptivity after ovarian stimulation for in vitro fertilization: a prospective randomized trial comparing fresh and frozen-thawed embryo transfers in high responders. Fertil Steril. 2011;96:516-8.

69. Shapiro BS, Daneshmand ST, Garner FC, Aguirre M, Hudson C, Thomas S. Evidence of impaired endometrial receptivity after ovarian stimulation for in vitro fertilization: a prospective randomized trial comparing fresh and frozenthawed embryo transfer in normal responders. Fertil Steril. 2011;96:344-8.

70. Griesinger G, Kolibianakis EM, Papanikolaou EG, Diedrich K, Van Steirteghem A, Devroey P, et al. Triggering of final oocyte maturation with gonadotropin-releasing hormone agonist or human chorionic gonadotropin. Live birth after frozen-thawed embryo replacement cycles. Fertil Steril. 2007;88:616-21.

71. Griesinger G, Schultz L, Bauer T, Broessner A, Frambach T, Kissler S. Ovarian hyperstimulation syndrome prevention by gonadotropin-releasing hormone agonist triggering of final oocyte maturation in a gonadotropin-releasing hormone antagonist protocol in combination with a "freeze-all" strategy: a prospective multicentric study. Fertil Steril 2011;95:2029, 33, 2033.e1.

72. Devroey P, Polyzos NP, Blockeel C. An OHSS-Free Clinic by segmentation of IVF treatment. Hum Reprod. 2011;26:2593-7.
73. Nachtigall RD, Mac Dougall K, Lee M, Harrington J, Becker G. What do patients want? Expectations and perceptions of IVF clinic information and support regarding frozen embryo disposition. Fertil Steril. 2010;94:2069-72.

74. Heng BC. Oocyte cryopreservation as alternative to embryo cryopreservation-some pertinent ethical concerns. Reprod Biomed Online. 2007;14:402-3.

75. Groenewoud ER, Cantineau AE, Kollen BJ, Macklon NS, Cohlen BJ. What is the optimal means of preparing the endometrium in frozen-thawed embryo transfer cycles? A systematic review and meta-analysis. Hum Reprod Update. 2013;19:458-70.

76. Ghobara T, Vandekerckhove P. Cycle regimens for frozen-thawed embryo transfer. Cochrane Database Syst Rev 2008;(1):CD003414. doi:CD003414.

77. Atkinson P, Koch J, Ledger WL. GnRH agonist trigger and a freeze-all strategy to prevent ovarian hyperstimulation syndrome: a retrospective study of OHSS risk and pregnancy rates. Aust N Z J Obstet Gynaecol. 2014;54:581-5.

78. Borges Jr E, Braga DP, Setti AS, Vingris LS, Figueira RC, Iaconelli Jr A. Strategies for the management of OHSS: results from freezing-all cycles. J Bras Reprod Assist. 2016;20:8-12.

79. Celada P, Bosch E, De Los Santos MJ, Giles J, Pellicer A. Elective frozen embryo transfer does not improve reproductive outcome in normo-responder patients. Hum Reprod. 2015;30 Suppl 1:i112.

80. Roque M, Valle M, Guimaraes F, Sampaio M, Geber S. Freeze-all policy: fresh vs. frozen-thawed embryo transfer. Fertil Steril. 2015;103:1190-3. 